STUDI

FRANCESI

\section{Studi Francesi}

Rivista quadrimestrale fondata da Franco Simone

153 (LI | III) | 2007

L'UMANESIMO IN EUROPA In ricordo di Franco Simone

\title{
Enrica Salvaneschi, Giardini ospitali. Ambienti e momenti di Emile Zola poeta
}

\section{Nicola Ferrari}

\section{(2) OpenEdition}

1 Journals

\section{Edizione digitale}

URL: http://journals.openedition.org/studifrancesi/9643

DOI: 10.4000/studifrancesi.9643

ISSN: 2421-5856

\section{Editore}

Rosenberg \& Sellier

\section{Edizione cartacea}

Data di pubblicazione: 1 décembre 2007

Paginazione: 691-692

ISSN: 0039-2944

\section{Notizia bibliografica digitale}

Nicola Ferrari, «Enrica Salvaneschi, Giardini ospitali. Ambienti e momenti di Emile Zola poeta», Studi

Francesi [Online], 153 (LI | III) | 2007, online dal 30 novembre 2015, consultato il 13 janvier 2021. URL: http://journals.openedition.org/studifrancesi/9643 ; DOI: https://doi.org/10.4000/studifrancesi.9643

Questo documento è stato generato automaticamente il 13 janvier 2021.

\section{(c) (i) $\odot$}

Studi Francesi è distribuita con Licenza Creative Commons Attribuzione - Non commerciale - Non opere derivate 4.0 Internazionale. 


\title{
Enrica Salvaneschi, Giardini ospitali. Ambienti e momenti di Emile Zola poeta
}

\author{
Nicola Ferrari
}

\section{NOTIZIA}

ENRICA SALVANESCHI, Giardini ospitali. Ambienti e momenti di Emile Zola poeta, Castel

Maggiore, Book, 2006, pp. 324.

1 Questo libro è certamente molti libri, ma soprattutto due. Il primo costruisce un autentico romanzo critico intorno al tema del giardino (ospitale, nel senso leopardiano di ospedale, ricetto di sofferenza, asilo agli atroci fantasmi di bellezza, insopportabili ferite nello scontro vivente e continuo di violenza e disinganno). Il tema, dall'accezione propria al suo slittamento metonimico, viene scandagliato nelle possibili variazioni figurali (del giardino cimitero, del giardino Eden, della vita vegetale specchio dell'umana esistenza - tra donne fiore e uomini alberi), accostato in polifoniche associazioni semantiche (con la neve, il fuoco, la capigliatura), condotto in raffinate concatenazioni modulanti a più lontane regioni (la sua tensione ascensionale conduce al tema dell'altalena, quindi alla bambola, alla donna-capra). All'interno del vasto affresco sinfonico dei Rougon-Macquart, l'indagine - tesa a scoprire ogni affiorare del tema, ogni baluginio (per quanto marginale, digressivo, episodico) - permette di rileggere differentemente anche i luoghi che già avremmo immaginato topici (su tutti, probabilmente, l'innervante, estenuato, simbolismo vegetale della Faute), scoprendo e raccontando in essi la tensione strutturale con l'insieme, l'esodo di rarefazioni, slittamenti, ampliamenti da un volume all'altro del ciclo, la fitta trama di rimandi, derive e rispondenze, espressione della forza creativa e della particolare interpretazione offerta da Zola agli archetipi letterari. L'attualità documentaria oltranzisticamente rivendicata dalla narrazione romanzesca zoliana rivela una profonda, sotterranea, esigenza mitica: prescindendo dal canto - melodrammatico e noto, immediatamente udibile e udito, dell'impegnata interpretazione del presente -, l'analisi (ma sarebbe meglio ribadire: il racconto analitico) permette di ascoltarne il 
fitto sostegno armonico - nell'inesausto confronto rielaborativo e trasfigurativo di materiali classici e cristiani. Attraverso l'appassionata (e appassionante) osservazione delle derive tematiche (percorsa dal fremito di continui riconoscimenti - lo sguardo nella moderna fattura del tappeto coglie figure antiche, Fedra, Tantalo, Sisifo), dimostrando una tracimante letterarietà della scrittura, si vuole eradicare l'intenzionale immagine naturalista dello Zola fotografico e documentale - dalle sue dichiarazioni di poetica trasferita di peso alla vulgata scolastica - rivendicando la più complessa dimensione simbolista di uno Zola scrittore, maestro di stile e costruzione permettendo di leggervi, dall'angolata prospettiva dei suoi giardini, quanto vi aveva intuito, in generale, Valéry: dietro l'accumulateur da lavorazione grossolana (i cui materiali vogliono emergere, ideologicamente, nella loro naturalità non sofisticata, non mediata - di denuncia, di urlo, brutalità senza alcuna trasmutazione, sans alchimie), Zola rappresenta il solo romanziere che avesse (quasi una qualità intrinseca, non perseguita, della sua scrittura) la 'composition musicale'. Svolto come atto di conquistata ammirazione, l'impegno a restituire la complessa e composta testura motivica del cosmo narrativo zoliano ne proietta la trama nell'universo di una Letteratura davvero goethianamente universale, assaporando innesti genetici e progressioni simboliche, trapassi, evoluzioni e contaminazioni da Omero, Euripide, Pindaro, Saffo, Longo Sofista, Virgilio, Tertulliano, Petrarca, Tasso, Leopardi, Kleist, Jean Paul, Andersen, Scott, Goncourt, Prus, Cvetaeva. Con l'eccezione del caso di Fontane, la folgorante sequenza di incontri testuali inusitati (vertiginosi negli allacci temporali e nei ponti geografici, sperimentazione delle più suggestive ipotesi $\mathrm{di}$ affinità) prescinde programmaticamente dai documentabili rapporti storici di influenza: gli accostamenti vengono riconosciuti, infatti, da Salvaneschi «tanto più cogenti quanto meno intenzionali, perché in qualche modo imputabili alla forza propulsiva che un archetipo assume nella diaspora dello spazio e del tempo, quando trova a recepirlo l'oggetto e il soggetto geniale». L'appassionato esercizio critico, liberato dai condizionamenti documentaristici, acquisisce una personale dimensione di ascolto, tesa a cogliere (e riprodurre) segrete risonanze, consonanze, vibrazioni armoniche dei testi nei testi, in ideale, permanente, compresenza. L'evocazione storica di un autore sembra trapassare irresistibilmente in celebrazione mitica del Letterario - universo brunianamente infinito che, in quanto tale, perde centri e periferie, ma manifesta la sua sostanza in ogni frammento. La libera esplorazione di questo universo, affidata all'intuizione, alla sensibilità, alla creatività dell'interprete (della sua individuale memoria culturale, del suo gusto associativo di nessi e relazioni), accetta scientemente l'azzardo metodologico e rilancia l'atto critico in gesto poetico.

2 Entro le più intime ragioni di questo primo libro, si può leggere il secondo. La grande pagina d'amore che Salvaneschi dedica con questo suo libro a Zola - consegna imperiosa di leggere e rileggere (à bout de souffle) - germina da un profondo riconoscimento stilistico: che "alcuni brani dei suoi romanzi siano pervasi da un'urgente qualità poetica che l'autore sa ben rendere riconoscibile nel ritmo della sua prosa». Quella poesia che avevamo inteso come manifestazione musicale (polifonica, variativa, ricorrente, modulante) dei temi, la poematicità strutturale e macroscopica, sinfonica, dell'insieme si riflette nello stesso andamento della pagina, nell'incedere ritmico della frase, nell'improvviso coagularsi della prosa in verso. L'intenzione di lettura si concreta arditamente in pratica di scrittura: disgregati e ricomposti dalla narrazione critica - di dispersione centripeta e proiezione centrifuga, degli archetipi - $\mathrm{i}$ passi dei Rougon accolti nei Giardini ospitali, vengono tradotti in un vibrante, plastico 
verso lungo italiano - "senza obblighi né preclusioni di rima", con rivisitazioni di strutture tradizionali come elegia, madrigale e sestina - per esplicitare le qualità più segrete (e poter così intendere letteralmente la menzione in sottotitolo) dell'Emile Zola poeta. Nella sua doppia declinazione, la realizzata vocazione della critica come invenzione e ricreazione poetica garantisce ai due libri (ma molti altri si possono leggere nelle pieghe di questo Saggio) unità profonda di slancio e respiro. 